\title{
Is early detection of late-onset Pompe disease a pneumologist's affair? A lesson from an Italian screening study
}

\author{
Marco Confalonieri ${ }^{1,11^{*}}$ (D), Michele Vitacca ${ }^{2}$, Raffaele Scala ${ }^{3}$, Mario Polverino ${ }^{4}$, Eugenio Sabato ${ }^{5}$, \\ Grazia Crescimanno ${ }^{6}$, Piero Ceriana ${ }^{7}$, Caterina Antonaglia', Gabriele Siciliano ${ }^{8}$, Nadja Ring ${ }^{9}$, Serena Zacchigna ${ }^{9}$, \\ Francesco Salton ${ }^{1}$, Andrea Vianello ${ }^{10}$ and on behalf of AIPO Pneumoloped Group
}

\begin{abstract}
Background: Late-onset Pompe disease (LOPD) is a recessive disease caused by a-glucosidase (GAA) deficiency, leading to progressive muscle weakness and/or respiratory failure in children and adults. Respiratory derangement can be the first indication of LOPD, but the diagnosis may be difficult for pneumologists. We hypothesize that assessing the GAA activity in suspected patients by a dried blood spot (DBS) may help the diagnosis of LOPD in the pneumological setting.

Population and methods: We performed a multicenter DBS survey of patients with suspected LOPD according to a predefined clinical algorithm. From February 2015 to December 2017, 140 patients (57 \pm 16 yrs., 80 males) were recruited in 19 Italian pneumological units. The DBS test was performed by a drop of blood collected on absorbent paper. Patients with GAA activity $<2.6 \mu \mathrm{mol} / \mathrm{L} / \mathrm{h}$ were considered positive. A second DBS test was performed in the patients positive to the first assay. Patients testing positive at the re-test underwent a skeletal muscle biopsy to determine the GAA enzymatic activity.

Results: 75 recruited subjects had outpatient access, 65 subjects were admitted for an acute respiratory failure episode. Two patients tested positive in both the first and second DBS test (1.4\% prevalence), and the LOPD diagnosis was confirmed through histology, with patients demonstrating a deficient GAA muscle activity (3.6 and $9.1 \mathrm{pmol} / \mathrm{min} / \mathrm{mg}$ ). A further five subjects were positive in the first DBS test but were not confirmed at re-test. The two positive cases were both diagnosed after hospitalization for acute respiratory failure and need of noninvasive ventilation. Most of the recruited patients had reduced maximal respiratory pressures (MIP $50 \pm 27 \%$ and MEP $55 \pm 27 \%$ predicted), restrictive pattern ( $\left.\mathrm{FEV}_{1} / \mathrm{FVC} 81.3 \pm 13.6\right)$ and hypoxaemia $\left(\mathrm{PaO}_{2} 70.9 \pm 14.5 \mathrm{mmHg}\right)$. Respiratory symptoms were present in all the patients, but only $48.6 \%$ of them showed muscle weakness in the pelvic girdle and/ or in the scapular girdle (35.7\%).
\end{abstract}

Conclusions: DBS GAA activity test may be a powerful screening tool among pneumologists, particularly in the acute setting. A simple clinical algorithm may aid in the selection of patients on which to administer the DBS test.

Keywords: Late-onset Pompe disease, Acute respiratory failure, Respiratory high dependency care unit, Noninvasive ventilation, Diagnosis

\footnotetext{
* Correspondence: marco.confalonieri@asuits.sanita.fvg.it

${ }^{1}$ Pneumology Unit, Dept. of Medical, Surgical and Health Sciences, University

of Trieste, Trieste, Italy

${ }^{11}$ Pulmonology Unit, University Hospital of Cattinara, Strada di Fiume 447,

34149 Trieste, Italy

Full list of author information is available at the end of the article
}

(c) The Author(s). 2019 Open Access This article is distributed under the terms of the Creative Commons Attribution 4.0 International License (http://creativecommons.org/licenses/by/4.0/), which permits unrestricted use, distribution, and reproduction in any medium, provided you give appropriate credit to the original author(s) and the source, provide a link to the Creative Commons license, and indicate if changes were made. The Creative Commons Public Domain Dedication waiver (http://creativecommons.org/publicdomain/zero/1.0/) applies to the data made available in this article, unless otherwise stated. 


\section{Introduction}

Pompe disease (ORPHA\#365) is a rare autosomal recessive disease due to alpha-glucosidase (GAA) deficiency, leading to glycogen accumulation in multiple tissues with a predilection for the skeletal muscle [1]. Depending on the age of onset, two different clinical forms have been described: infantile and late-onset [2]. Late-onset Pompe disease (LOPD) is a slowly progressive form associated with a residual enzyme activity, which presents with either juvenile or adult onset and shows various clinical phenotypes $[3,4]$.

Early clinical manifestations of LOPD are usually progressive muscle weakness and/or respiratory failure [5]. In contrast to what happens in other hereditary neuromuscular diseases, in which respiratory failure occurs after the loss of ambulation, respiratory involvement in LOPD may represent the first clinical manifestation of the disease itself, so that patients may have respiratory disorders despite retaining ambulation [6]. Approximately one third of adult patients affected by Pompe disease have an early respiratory phenotype, with a clinical picture that includes dyspnea and/or respiratory failure, sleep-disordered breathing (SDB) and recurrent pulmonary infections [6]. Acute respiratory failure requiring mechanical ventilation in Intensive Care Units (ICU) or in Respiratory High Dependency Care Units (RHDCU) may be the first clinical presentation of the disease [7]. However, LOPD with a prevalent respiratory derangement is not easily and promptly identified during an acute respiratory failure episode because the critical illness itself doesn't allow a clearly diagnostic electromyographic study [8].

Enzyme replacement therapy (ERT) with alglucosidase alpha was approved for LOPD because it can stabilize lung function and improve walking distance [9]. Moreover, ERT may remarkably reduce muscle lysosomal glycogen [10] and also the mortality rate compared to untreated patients [11]. Therefore, a timely diagnosis and establishment of ERT is associated with maximized clinical benefit [12].

Recently, the simple measurement of GAA activity in a dried blood spot (DBS) was proposed as a screening method [13]. We hypothesize that the pneumologists could play a pivotal role in the diagnosis of LOPD by applying the DBS technique. Thus, we organized a national DBS-based screening study in the pneumological field.

\section{Methods}

The Italian Association of Hospital Pneumologists (AIPO) selected 19 pneumological centres distributed in almost every region of Italy with recognized experience in the management of patients affected by neuromuscular disorders and respiratory derangement.
We performed a multicenter DBS-based case-finding study of consecutive patients with suspected LOPD according to a predefined clinical algorithm (Fig. 1), as determined during the pneumological visit or admission to the respiratory unit [14]. The inclusion criteria were: age $\geq 18$ yrs. and $<80$ yrs., the suspicion of a neuromuscular disorder with respiratory involvement according to $5+2$ items by Ambrosino et al. [14]:

1) Restrictive ventilatory deficiency (reduced FVC with normal $\mathrm{FEV}_{1} / \mathrm{FVC}$ )

2) Nightime hypoventilation ( $\mathrm{HbO} 2<90 \%$ for more than 5 consecutive minutes during cardiopulmonary monitoring

3) Hypoxemia and hypercapnia at ABG

4) Weakness of the respiratory muscles (MIP, MEP, PCEF)

5) Chest X-ray/CTscan/ultrasound significant for diaphragm palsy, atelectasis, etc.

6) Weakness of the scapular and/or pelvic girdle (facultative)

7) Increased blood creatin phosphokinase level (facultative).

At least five major criteria should be present for study inclusion, or alternatively four out of the first five major criteria plus at least one of the facultative criteria 6) and 7).

The exclusion criteria were: age $<18$ yrs. and $\geq 80$ yrs., presence of any already known neuromuscular disorder, previous or current ERT, presence of clinically apparent cardiac involvement, presence of conditions already explaining the inclusion criteria (e.g. heart failure, COPD ot chronic obstructive pulmonary disease, OSA or obstructive sleep apnea, overlap syndrome, post-surgical respiratory failure, post-ICU, hypoventilation-obesity syndrome, fibrothorax, diaphragm palsy of known origin, pulmonary fibrosis, endocrine diseases).

The DBS test was performed by half a drop of blood from a finger prick or from a venous blood sample. The blood drop was collected on absorbent paper and the enzyme activity was assessed in a diagnostic laboratory as previously described [15]. Patients with GAA activity $<2.6 \mu \mathrm{mol} / \mathrm{L} / \mathrm{h}$ were considered positive. A second DBS test (referred to as a re-test) was performed in all patients who resulted positive to the first assay. Positive patients at the re-test underwent a confirmatory step by determination of GAA enzymatic activity on skeletal muscle [2]. After biochemical confirmation, a molecular genetic analysis was performed by GAA gene sequencing to assess the genotype of patients with LOPD.

Ethical approval was given both centrally and at each individual centre. Patients gave written informed consent. All data are presented as mean \pm standard deviation 


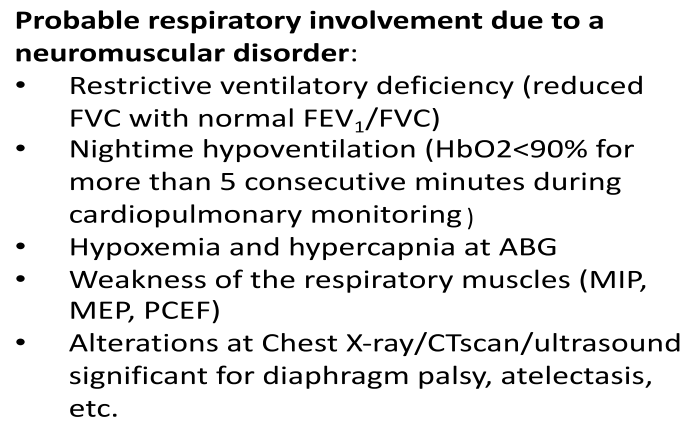

- Restrictive ventilatory deficiency (reduced FVC with normal FEV 1 /FVC)

- Nightime hypoventilation ( $\mathrm{HbO} 2<90 \%$ for more than 5 consecutive minutes during cardiopulmonary monitoring)

- Hypoxemia and hypercapnia at ABG

- Weakness of the respiratory muscles (MIP, MEP, PCEF)

- Alterations at Chest X-ray/CTscan/ultrasound significant for diaphragm palsy, atelectasis, etc.
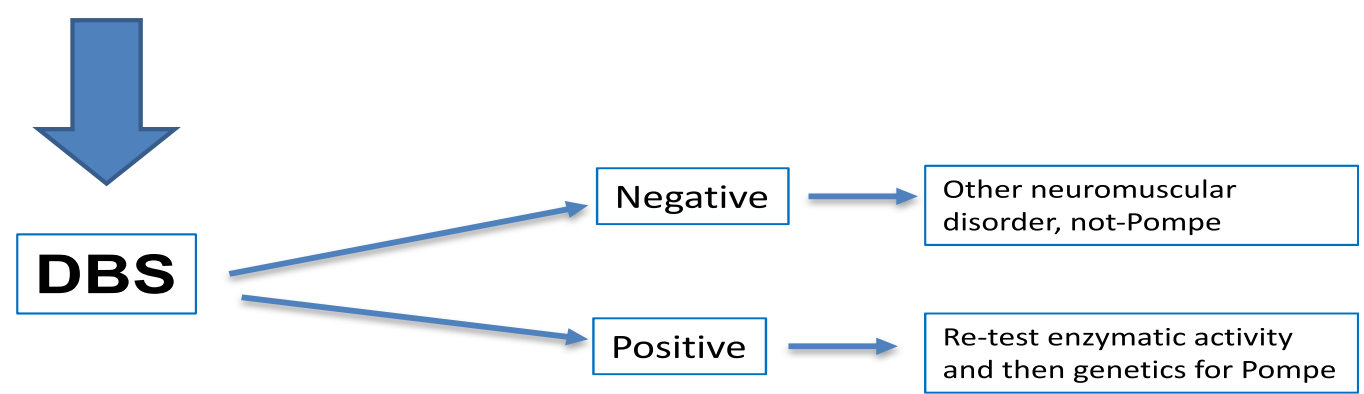

Fig. 1 Clinical algorithm to select patients for DBS test

(SD). Data analysis was performed using the GraphPad Prism version 6 software (San Diego, CA, USA). Data are presented as mean (SD) or median (min., max.), as appropriate. The positive and negative predictive values were computed from a $2 \times 2$ contingency table. The association between categorical variables was evaluated using Fisher's exact test. Differences between groups were assessed using the Student's t test.

\section{Results}

The study lasted from February 2015 to December 2017 and recruited 140 patients in 16 out of 19 Italian pneumological participating units with good experience in respiratory failure of neuromuscular origin. Two DBS-positive cases (patients positive in both the test and re-test) were found and confirmed to be LOPD. The cDNA mutations were respectively c.-32-13 T $>$ G (IVS1); c. $1564 \mathrm{C}>\mathrm{G}$ (p.Pro522Ala) and c.32-13 T > G; c.-673C > T. The characteristics of the recruited patients and the two LOPD confirmed patients are described in Table 1.

There were a further five subjects which tested positive in the first DBS test, but were not positive at the re-test. One of these cases could not be retested due to death, seemingly as a result of respiratory failure (the relatives did not authorized autopsy). In the studied population 80 patients were male and 60 were female; the median age at recruitment was 58 years (min.18max.86). The two positive cases were both diagnosed after hospitalization in RHDCU for an acute respiratory failure with need of noninvasive ventilation and cough assist devices, although they had reported symptoms (dyspnea on exertion, fatigue, sleep disturbances with somnolence during the day, upper and lower girdle weakness with an initial waddling gait and mild hyperlordotic lumbar spine) for at least one year before (mean $1.2 \pm 2$ ). Other 63 patients were recruited during hospital admission, while the remaining $75 \mathrm{pa}-$ tients underwent ambulatory pneumological visit for respiratory symptoms.

The muscle activity of GAA in the two LOPD patients were $3.6 \mathrm{pmol} / \mathrm{min} / \mathrm{mg}$ and $9.1 \mathrm{pmol} / \mathrm{min} / \mathrm{mg}$. Among the patients admitted to the hospital, 59 out of them required monitoring in a RHDCU, with need of noninvasive ventilation in 31 cases (52.5\%). All the recruited patients presented respiratory symptoms, and in fact, the most commonly reported symptoms by the recruited subjects were dyspnoea (121 patients, $86.4 \%$ of cases), fatigue (118 patients, $84.3 \%$ of cases), orthopnea (61 patients, $43.5 \%$ of cases), and further unspecific symptoms with overlapping frequency in our unconfirmed or positive patients. Most of the patients had reduced maximal respiratory pressures (MIP $50 \pm 27 \%$ predicted, MEP 55 $\pm 27 \%$ predicted), and restrictive pattern $\left(\mathrm{FEV}_{1} / \mathrm{FVC}\right.$ $81.3 \pm 13.6)$ with mild hypoxaemia $\left(\mathrm{PaO}_{2} 70.9 \pm 14.5\right.$ $\mathrm{mmHg}$ ). Less than half of the recruited subjects had mild to moderate muscular symptoms including weakness in the pelvic girdle (48.6\%) and/or in the scapular girdle (35.7\%). There was no adverse effect or delayed 
Table 1 Clinical characteristics of the patients

\begin{tabular}{|c|c|c|c|}
\hline Characteristics & Recruited patients & LOPD patient \# 1 & LOPD patient \#2 \\
\hline Gender, M/F & $80 \mathrm{M} / 60 \mathrm{~F}$ & M & $\mathrm{F}$ \\
\hline Age at recruitment, mean \pm SD & $57 \pm 16$ & 69 & 42 \\
\hline Months from symptoms onset, median (min, max) & $6(0-373)$ & 12 & 16 \\
\hline Body mass index, $\mathrm{kg} / \mathrm{m}^{2}$ & $28.5 \pm 9.5$ & 19.7 & 23.3 \\
\hline Respiratory symptoms & $100 \%$ & yes & yes \\
\hline - Dyspnea during exercise & $86.4 \%$ & yes & yes \\
\hline - Dyspnea at rest & $35.9 \%$ & no & yes \\
\hline - Ineffective cough & $41.1 \%$ & yes & yes \\
\hline - Ortopnea & $43.5 \%$ & no & no \\
\hline - Fatigue & $84.3 \%$ & yes & yes \\
\hline - Airways infections & $44.1 \%$ & no & yes \\
\hline Sleep disorders & $36.4 \%$ & yes & yes \\
\hline - Nocturnal restlessness & $44.2 \%$ & yes & yes \\
\hline - Frequent reawaken & $40.0 \%$ & no & yes \\
\hline - Nocturnal apnoea & $41.4 \%$ & no & no \\
\hline - Snoring & $30.0 \%$ & no & no \\
\hline - Morning sleepiness & $25.0 \%$ & no & yes \\
\hline - Morning headache & $20.0 \%$ & yes & no \\
\hline - Day sleepiness & $39.2 \%$ & yes & yes \\
\hline Acute respiratory failure at recruitment & $28.5 \%$ & yes & yes \\
\hline Myalgia & $52.1 \%$ & no & no \\
\hline CPKaemia, IU/L & $345 \pm 700$ & 206 & 471 \\
\hline AST, U/L & $27 \pm 13$ & 44 & 56 \\
\hline $\mathrm{PaCO} 2, \mathrm{mmHg}$ & $43 \pm 12$ & 54.2 & 46 \\
\hline Upright FVC \% predicted & $67 \pm 25$ & 62 & 66 \\
\hline$\Delta$ Upright-Supine FVC\% & $-18 \pm 20$ & -28 & -31 \\
\hline Lower-girdle muscle weakness, \% & $48.6 \%$ & yes & yes \\
\hline Upper-girdle muscle weakness, \% & $35.7 \%$ & yes & yes \\
\hline Walton\&Gardner-Medwin Scale & $2.9 \pm 3.1$ & 7 & 4 \\
\hline GAA activity, microMol/L/h & $10.1 \pm 6.6$ & 0.36 & 0.71 \\
\hline
\end{tabular}

$M$ males, $F$ females, CPK creatinphosphokinase, AST aspartate transaminase, PaCO2 arterial partial pressure carbon dioxide, FVC forced vital capacity, GAA alpha glucosidase

diagnosis due to the administration of the DBS GAA activity test. The prevalence of DBS+ subjects in our selected population was $4.2 \%$, while the prevalence of confirmed LOPD patients was1.4\%. No association between categorical variables was found. The sensitivity of DBS test in our population was $100 \%$, and the specificity $97.1 \%$. The positive predictive value (PPV) of the DBS test in the selected patient population was 0.333 (33.3\%), and the negative predictive value (NPV) was $1.000(100 \%)$.

\section{Discussion}

A timely diagnosis and treatment of LOPD is important to improve outcome [10], but latency from the onset of symptoms to an established diagnosis may be up to $5-$ 30 years from the onset of symptoms $[16,17]$. The delay of LOPD diagnosis is mainly due to the very low incidence (estimated 1 case in 57,000-100,000 in European countries) $[18,19]$, together with overlapping symptoms with other NMD [3, 18], but also the so-called "respiratory phenotype" might be a confounder [4]. Our national DBS-based screening study demonstrated that also in the pneumological setting it is possible to easily detect patients with undiagnosed LOPD after a patient selection by means of a dedicated clinical algorithm. Particularly, the late-onset form of glycogen storage disease type II or Pompe disease (LOPD) may be suspected in subjects with acute respiratory insufficiency, SDB and 
proximal muscle weakness without a clinically apparent cardiac involvement. In our study, both of the two patients found to have LOPD has displayed respiratory and neurologic symptoms for more than 1 year, but they were detected only during an episode of acute respiratory failure with the need of RHDCU admission. This latency of the diagnosis may seem too high, but it is much less than other literature reports $[8,16,17]$. In line with our results, Kishnani et al. [19] recently reported that patients with early respiratory involvement can be diagnosed sooner than those presenting with only muscular symptoms and/ or hyperCPKaemia.

Considering all the subjects included in our survey, most of them had outpatient access, while the other patients were admitted to the hospital with access to the emergency room (ER). Among the 65 patients admitted to the ER, most of them required monitoring in a RHDCU and noninvasive ventilatory support. This is concordant with the findings of the last Italian RHDCU survey, which highlighted the increased number of admissions for acute respiratory failure of neuromuscular origin as compared to the previous national census [20]. Nevertheless, most of the patients in our national screening study were recruited as outpatients visited by a pneumologist for a common symptom like exercise dyspnoea jointly with SDB and a suspected NMD. The execution of a DBS test for the detection of LOPD showed high sensitivity and specificity, without disturbing the correct diagnosis, or harming the patient. The re-test did not confirm four DBS+ subjects at the first test, but this did not influence the respiratory management. One of the two LOPD cases presented only slightly increased blood creatine phosphokinase (CPK) levels $(206 \mathrm{IU} / \mathrm{L})$, supporting the observation that CPK levels are almost normal in some cases of LOPD with respiratory phenotype without limb-girdle syndrome $[4,6$, 7]. Balancing the potential harms and benefits of diagnosing LOPD in the pneumological setting is not a contentious issue as there are disease-modifying treatments for Pompe disease, and a formal diagnosis may only benefit every patient.

\section{Conclusions}

Testing GAA activity by DBS was shown to be a powerful screening tool for pneumologists, particularly in the acute setting. A simple clinical algorithm may help the selection of patients to administer the DBS test in order to diagnose LOPD. Particular attention should be paid when a patient with suspected but undiagnosed NMD and acute respiratory failure without cardiac involvement needs mechanical ventilation and/or cough assist devices.

\section{Abbreviations}

ABG: Arterial blood gas; AIPO: Italian Association of Hospital Pneumologists; AST: Aspartate transaminase; COPD: Chronic obstructive pulmonary disease; CPK: Creatine phosphokinase; CTscan: Computed tomography scan; DBS: Dried Blood Spot; ER: Emergency Room; ERT: Enzyme replacement therapy; FEV1: Forced Expiratory Volume in the first second; FVC: Forced Vital Capacity; GAA: Alpha-glucosidase; ICU: Intensive Care Units; LOPD: Late-onset Pompe disease; MEP: maximal expiratory pressure; MIP: maximal inspiratory pressure; NMD: Neuromuscular disorder; OSA: Obstructive sleep apnea; PaCO2: Partial pressure carbon dioxide; PCEF: Peak cough expiratory flow; RHDCU: Respiratory High Dependency Care Units; SD: Standard deviation; SDB: Sleep-disturbed breathing

\section{Acknowledgements}

We thanks the other members of the AIPO Pneumoloped Group: Alessio Mattei (Torino), Fausto De Michele (Napoli), Luca Triolo (Roma), Giuseppe Culla (Roma), Pieraldo Canessa (Sarzana), Giuseppe Girbino (Messina), Mirco Lusuardi (Correggio), Enrico Perretta (Imperia), Claudio De Michelis (Imperia), Teresa Renda (Firenze).

\section{Funding}

Partial support of this study was derived from unrestricted grant by Sanofi Genzyme Italy.

\section{Availability of data and materials}

The datasets analyzed during the current study are available from the corresponding author on reasonable request.

\section{Authors' contributions \\ MC conceived-designed-coordinated the survey and drafted the manuscript; MV conceived the survey collected data and revised the manuscript, RF conceived the survey collected data and revised the manuscript, MP designed the survey collected data and revised the manuscript, ES collected data and helped to draft the manuscript, GC collected data and revised the final manuscript, PC collected data and revised the manuscript, CA collected data and drafted the manuscript, GS collected data and revised the manuscript, NR performed statistical analysis, reviewed English language, SZ cooperated to statistics and checked lab data, FS collected data and revised the manuscript, AV collected data and revised the manuscript. All authors read and approved the final manuscript.}

Ethics approval and consent to participate

Ethical approval was given by the C.E.R.U. Friuli-Venezia Giulia (ref.\# 67/2014).

Consent for publication

Not applicable.

\section{Competing interests}

The authors declare that they have no competing interests.

\section{Publisher's Note}

Springer Nature remains neutral with regard to jurisdictional claims in published maps and institutional affiliations.

\section{Author details}

'Pneumology Unit, Dept. of Medical, Surgical and Health Sciences, University of Trieste, Trieste, Italy. ${ }^{2}$ ICS S. Maugeri, Care and Research Institute, Respiratory Rehabilitation Unit, Lumezzane, Bs, Italy. ${ }^{3}$ Pneumology and Respiratory Intensive Care Unit, San Donato Hospital, Arezzo, Italy. ${ }^{4}$ Lung Diseases High Specialty Institute, Medical Sciences Department, Scafati, Salerno, Italy. "Pneumology Unit, "A. Perrino" General Hospital, Brindisi, Italy. ${ }^{6}$ Institute of Biomedicine and Molecular Immunology, Italian National Research Council, Palermo, Italy. ${ }^{7}$ ICS S. Maugeri, Care and Research Institute, Pulmonary Rehabilitation Unit, Pavia, Italy. ${ }^{8}$ Department of Clinical and Experimental Medicine, Neurology Unit, University of Pisa, Pisa, Italy. ${ }^{9}$ International Centre for Genetic Engineering and Biotechnology, Trieste, Italy. ${ }^{10}$ Respiratory Pathophysiology and Intensive Care Unit, Department of Cardio-Thoracic, University-City Hospital of Padova, Padova, Italy.

${ }^{11}$ Pulmonology Unit, University Hospital of Cattinara, Strada di Fiume 447, 34149 Trieste, Italy. 
Received: 12 November 2018 Accepted: 21 February 2019

Published online: 04 March 2019

\section{References}

1. Raben N, Plotz P, Byrne BJ. Acid-alpha-glucosidase deficiency (glycogenosis type II, Pompe disease). Curr Mol Med. 2002;2:145-66.

2. Kishnani PS, Steiner RD, Bali D, Berger K, Byrne BJ, Case LE, et al. Pompe disease diagnosis and management guidelines. Genetics Med. 2006:8:267-88.

3. Manganelli F, Ruggiero L. Clinical features of Pompe disease. Acta Myol. 2013;32:82-4.

4. Chan J, Desai AK, Kazi ZB, Corey K, Austin S, Hobson-Webb LD, et al. The emerging phenotype of late-onset Pompe disease: a systematic literature review. Mol Gen Metabol. 2017;120:163-72.

5. Winkel LP, Hagemans ML, Van Doorn PA, Loonen MC, Hop WJ, Reuser AJ, et al. The natural course of non-classic Pompe's disease; a review of 225 published cases. J Neurol. 2005;252:875-84.

6. Mellies U, Lofaso F. Pompe disease: a neuromuscular disease with respiratory muscle involvement. Respir Med. 2009;103:477-84.

7. Keunen RWM, Lambregts PCLA, Op de Coul AAW, Joosten EMG. Respiratory failure as initial symptom of acid maltase deficiency. J Neurol Neurosurg Psychiatry. 1984;47:549-52.

8. Menzella F, Codeluppi L, Lusuardi M, Galeone C, Valzania F, Facciolongo N. Acute respiratory failure as presentation of late-onset Pompe disease complicating the diagnostic process as a labyrinth: a case report. Multidiscip Respir Med. 2018;13:32

9. Van der Ploeg AT, Clemens PR, Corzo D, Escolar DM, Florence J, Groeneveld GJ, et al. A randomized study of alglucosidase alpha in late-onset Pompe's disease. N Engl J Med. 2010;362:1396-406.

10. Cupler EJ, Berger KI, Leshner RT, Wolfe Gl, Han JJ, Barhon NJ, et al. Consensus treatment recommendations for late-onset Pompe disease. Muscle Nerve. 2012;45:319-33.

11. Schoser B, Stewart A, Kanters S, Hamed A, Jansen J, Chan K, et al. Survival and long-term outcomes in late-onset Pompe disease following alglucosidase alfa treatment: a systematic review and meta-analysis. J Neurol. 2017;264:621-30.

12. van der Ploeg A, Carlier PG, Carlier RY, Kissel JT, Schoser B, Wenninger S, et al. Prospective exploratory muscle biopsy, imaging, and functional assessment in patients with late-onset Pompe disease treated with alglucosidase alfa: the EMBASSY study. Mol Genet Metab. 2016;119:115-23.

13. Musumeci O, la Marca G, Spada M, Mondello S, Danesino C, Comi GP, et al. LOPED study: looking for an early diagnosis in a late-onset Pompe disease high-risk population. J Neurol Neurosurg Psychiatry. 2016;87:5-11.

14. Ambrosino N, Confalonieri M, Crescimanno G, Vianello A, Vitacca M. The role of respiratory management of Pompe disease. Respir Med. 2013;107: 1124-32.

15. Zhang H, Kallwas H, Young SP, Carr C, Dai J, Kishnani PS, et al. Comparison of maltose and acarbose as inhibitors of maltase-glucoamylase activity in dried blood spots for the diagnosis of infantile Pompe disease. Genet Med Dis. 2006:8:302-6.

16. Hagemans MLC, Winkel LPF, Van Doorn PA, Hop WJ, Loonen MC, Reuser AJ, et al. Clinical manifestation and natural course of late-onset Pompe's disease in 54 Dutch patients. Brain. 2005;128(Pt 3):671-7.

17. Muller-Felber W, Horvath R, Gempel K, Podskarbi T, Shin Y, Pongratz D, et al. Late onset Pompe disease: clinical and neurophysiological spectrum of 38 patients including long- term follow-up in 18 patients. Neuromuscul Disord. 2007;17:698-706.

18. American Association of Neuromuscular \& Electrodiagnostic Medicine. Diagnostic criteria for late-onset (childhood and adult) Pompe disease. Muscle Nerve. 2009;40:149-60.

19. Kishnani PS, Amartino HM, Lindberg C, Miller TM, Wilson A, Keutzer J. Timing of diagnosis of patients with Pompe disease: data from the Pompe registry. Am J Genet A. 2013;161:2431-43.

20. Scala R, Corrado A, Confalonieri $M$, et al. Increased number and expertise of Italian respiratory high-dependency care units: the second national survey. Respir Care. 2011;56:1100-7.

Ready to submit your research? Choose BMC and benefit from:

- fast, convenient online submission

- thorough peer review by experienced researchers in your field

- rapid publication on acceptance

- support for research data, including large and complex data types

- gold Open Access which fosters wider collaboration and increased citations

- maximum visibility for your research: over $100 \mathrm{M}$ website views per year

At BMC, research is always in progress.

Learn more biomedcentral.com/submissions 